Revue d'histoire de l'Amérique française

ZAS REVUE D.HISTOIRE DE L'AMÉRIQUE FRANÇAISE

\title{
Ludger Duvernay et les débuts de la presse périodique aux Trois-Rivières
}

Yves Tessier

Volume 18, numéro 4, mars 1965

URI : https://id.erudit.org/iderudit/302435ar

DOI : https://doi.org/10.7202/302435ar

Aller au sommaire du numéro

Éditeur(s)

Institut d'histoire de l'Amérique française

ISSN

0035-2357 (imprimé)

1492-1383 (numérique)

Découvrir la revue

\section{Citer ce document}

Tessier, Y. (1965). Ludger Duvernay et les débuts de la presse périodique aux Trois-Rivières. Revue d'histoire de l'Amérique française, 18(4), 624-627.

https://doi.org/10.7202/302435ar d'utilisation que vous pouvez consulter en ligne.

https://apropos.erudit.org/fr/usagers/politique-dutilisation/ 


\section{BIBLIOGRAPHIE *}

\section{LUDGER DUVERNAY ET LES DÉBUTS DE LA PRESSE PERRIODIQUE AUX TROIS-RIVIERES}

$$
\text { I - SOURCES }
$$

A - SOURCES MANUSCRITES

Archives de la Province de Québec

Papier Duvernay, les pièces 1 à 62 concernent la vie de Ludger Duvernay, de 1799 à 1826.

Archives du Séminaire Saint-Joseph des Trois-Rivières

Papiers Trifluviens, colligés par Monseigneur Albert Tessier. Les quelques rares pièces manuscrites de Duvernay sont des reçus, des états de compte.

Archives de la Ville des Trois-Rivières

Documents relatifs à la Société $d u$ Feu, en tant qu'inspecteur des incendies, Duvernay a signé quelques inventaires de matériel de protection contre le feu.

\section{B - SOURCES IMPRIMÉES}

\section{1 - Journaux de Duvernay}

L'Ami de la religion et du Roi, journal ecclésiastique, politique et littéraire. Trois-Rivières, imprimé par Ludger Duvernay, juin 1820 - 1821 ? 16 p. par numéro, pagination continue. $17 \mathrm{~cm}$. Mensuel. (Les numéros 2 et 4 , les seuls consultés, sont conservés à la bibliothèque Saint-Sulpice, à Montréal).

L'Argus, journal électorique. Trois-Rivières, imprimé et publié par Ludger Duvernay, 30 août 1826 - 30 novembre 1826. 4 p. par numéro, non paginé. $37.5 \mathrm{~cm}$. Hebdomadaire.

* Voir l'article de M. Yves Tessier, sur ce sujet, aux pages de notre Revue, déc. 1964: 387-404 et 566-581 de ce numéro de mars 1965 . 
Le Constitutionnel, gazette française des Trois-Rivières, journal politique et littéraire. Trois-Rivières, imprimé et publié par Ludger Duvernay, 11 mars 1823 - 1825 ?. 4 p. par numéro, non paginé. $32.5 \mathrm{~cm}$. Hebdomadaire.

La Gazette des Trois-Rivières. Trois-Rivières, imprimé et publié par Ludger Duvernay, 12 août 1817 - 1821 ? . 4 p. par numéro, non paginé. $43 \mathrm{~cm}$. Hebdomadaire.

\section{2 - Autres journaux}

Le Canadien. Québec, Bédard, 22 novembre 1806 - 17 mars 1810 ; Bédard, 14 juin 1817 - 15 décembre 1819; Vallerand, 19 janvier 1820 - 2 mars $1825 \ldots$

Le Spectateur canadien. Montréal, imprimé et publié par CharlesBernard Pasteur, 1815. 4 p. par numéro, non paginé. $44 \mathrm{~cm}$. Hebdomadaire.

(Sauf indication contraire, ces journaux ont été consultés à la Bibliothèque générale de l'Université Laval).

\section{II - INVENTAIRES}

- - Rapport de l'Archiviste de la Province de Québec pour 1926-1927. L. Amable Proulx, imprimeur de sa Majesté le Roi, 1927. 434 p. $25 \mathrm{~cm}$. Un relevé complet des Papiers Duvernay est fait dans les pages 147 à 252. Le contenu de chaque pièce est résumé dans une brève note.

$$
\text { III - ETUDES }
$$

\section{A - Études spéciales}

Auger, Roland. Essai de bio-bibliographie sur Ludger Duvernay, Imprimeur, journaliste et fondateur de la Société Saint-JeanBaptiste. Montréal, Ecole de bibliothéconomie de l'Université de Montréal, 1953. 114 p. (Thèse sur microfilm conservé à la Bibliothèque de la Législature). Ouvrage excellent. L'auteur ne fait pas mention cependant des pièces manuscrites conservées aux archives de la Ville des TroisRivières.

Fréchette, L.-A. Ludger Duvernay, fondateur de la Société SaintJean-Baptiste, dans l'Ecrin, s. 1.n.d. 63 p. $21 \mathrm{~cm}$. Quelques notes biographiques très brèves. 
Gagner, J.-Léopold. Duvernay et la Saint-Jean-Baptiste. Montréal, Chanteclerc, c1952. 52 p. $19 \mathrm{~cm}$. On met en évidence surtout le rôle de Duvernay parmi les Patriotes.

Giraldi, Auguste. Ludger Duvernay. Brooklyn, s.ed. 1922. 11 p. $19 \mathrm{~cm}$. Biographie très succincte de Duvernay.

Parent, Roger-D. Duvernay, le Magnifique. Montréal, Institut de la Nouvelle-France, 1943. 32 p. $19 \mathrm{~cm}$. Ouvrage bref, avec un portrait de Duvernay. On retrouve l'essentiel de la vie de Ludger Duvernay.

\section{B - ÉtUdes DIVERSES}

Bonenfant, Jean-Charles. Inventaire des sources, les journaux, dans Recherches sociographiques, v. II, no 3-4, juillet-décembre 1961.

Bovey, Wilfrid. Les Canadiens-Français d'aujourd'hui, l'essor d'un peuple. Traduit de l'anglais par Jean-Jacques Lefebvre. Montréal, Editions de l'ACF, 1940. 418 p. $23 \mathrm{~cm}$.

Dionne, Narcisse-Eutrope. Les Ecclésiastiques et les royalistes français réfugiés au Canada à l'époque de la Révolution, 1791-1802. Québec, s.ed., 1905. 447 p. $21.5 \mathrm{~cm}$.

Dionne, Narcisse-Eutrope. Inventaire chronologique des livres, brochures, journaux et revues publiés en langue française dans la province de Québec, depuis l'établissement de l'imprimerie au Canada jusqu'à nos jours, 1764-1905. Québec, s.ed., 1905.4 v. $26 \mathrm{~cm}$.

Gagnon, Philéas. Essai de bibliographie canadienne, inventaire d'une bibliothèque comprenant imprimés, manuscrits, estampes, etc., relatifs à l'histoire du Canada et des pays adjacents, avec les notes bibliographiques. Québec, s.ed., 3 t. $25 \mathrm{~cm}$.

Groulx, Lionel. Notre maître le passé. Montréal, Granger, 1944. 319 p. $18 \mathrm{~cm}$.

Hatin, Eugène. Bibliographie historique et critique de la Presse périodique française. Paris, Firmin Didot, 1866. 660 p. 23.5 $\mathrm{cm}$. Volume tiré par procédé photographique en 1960. (Bibliothèque de l'Université Laval).

Lareau, Edmond. Mélanges historiques et littéraires. Montréal, Eusèbe Sénécal, 1877. 352 p. $17 \mathrm{~cm}$. 
Lessard, Claude. Histoire de l'éducation au Séminaire de Nicolet, 1803-1863. Thèse de diplôme d'Etudes Supérieures, Université Laval, juin 1963.

Roy, Pierre-Georges. La famille Rocbert de la Morandière. Lévis, s.ed., 1905.88 p. $23 \mathrm{~cm}$.

Sulte, Benjamin. "Anciens journaux des Trois-Rivières". Bulletin des Recherches historiques, v. 7, 1901.

Sulte, Benjamin. La Saint-Jean-Baptiste, 1636-1852. Publié par Gérard Malchelosse. Montréal, Edouard Garand, 1929. (Mélanges historiques, v. 15). 130 p. $24 \mathrm{~cm}$.

Tessier, Albert. Trois-Rivières, 1535-1935. Trois-Rivières, Le Nouvelliste, 1935.199 p. $25 \mathrm{~cm}$.

Trudel, Marcel. L'influence de Voltaire sur les écrivains francais du Canada, de 1760 à 1900. Thèse pour le doctorat ès Lettres, mai 1945. $494 \mathrm{p}$.

Les Ursulines des Trois-Rivières depuis leur établissement jusqu'à nos jours. Trois-Rivières, P. V. Ayotte, 4 t. $21 \mathrm{~cm}$.

Vallée, Henri. Journaux et journalistes d'autrefois. Dans Le Nouvelliste, 10 juillet 1948.

Vallée, Henri. Les journaux trifluviens de 1817 à 1933. TroisRivières, le Bien Public, 1933. (Pages trifluviennes, série A, no 6). 92 p. $24.5 \mathrm{~cm}$.

Têtu, Horace. Historique des journaux de Québec. Québec, s.ed., 1889. $107 \mathrm{p} .19 \mathrm{~cm}$.

Wright, George F. Journalism, dans William Wood, The Storied Province of Quebec, Past and Present. Toronto, The Dominion Publishing Company, 1931. 4 v. $28 \mathrm{~cm}$.

YVES TESSIER, bachelier ès arts, bachelier en histoire. 